\title{
ON CATALECTICANT PERFECT IDEALS OF CODIMENSION 2
}

\author{
Zaqueu Ramos 1 Aron Simis 2
}

\begin{abstract}
One deals with catalectic codimension two perfect ideals and certain degenerations thereof, with a view towards the nature of their symbolic powers. In the spirit of [10] one considers linearly presented such ideals, only now in the situation where the number of variables is sufficiently larger than the size of the matrix, yet still stays within reasonable bounds.
\end{abstract}

\section{Introduction}

The idea in this work is to consider a codimension 2 perfect ideal $I$ in a polynomial $\operatorname{ring} R=$ $k\left[X_{1}, \ldots, X_{n}\right]$ ( $k$ a field) whose $m \times(m-1)$ presentation matrix is a generic catalecticant or a degeneration of this in a sense to be explained. We examine three of these constructs: catalecticants with leap, sub-Hankel and semi-Hankel matrices. Depending on the values of $n$ and $m$ in the sub-Hankel case the ring $R / I$ may fail to be a normal domain (this is to be compared to with [10, Proposition 2.3], where normality always holds if $n \geq 4$ ). As it turns out, a special instance of the semi-Hankel case leads to a Cremona transformation. Such a Cremona transformation does not seem to have been observed before in this systematization.

As a rule, the sort of results obtained for all the above constructs have to do with the properties of the ideals of minors, lower order minors as well. Since mostly $m<<n$, there is room enough to ask whether $I$ is of linear type or at least locally so on the punctured spectrum, typically via variations of the so-named $\left(G_{s}\right)$ condition. The other property of interest is normally tosionfreeness. Mostly, $I$ will be normally torsionfree, but there are some exceptions. It becomes clear that whether this property holds has to do with the nature of the degeneration of the catalecticant (or the generic matrix).

Since basically every section deals with one of the constructs and there is essentially one theorem in each case, there is hardly any point in enlarging this foreword with further details. As a way of compensation, we will expand on the terminology and tools used throughout.

For the proofs we have drawn quite a bit on the results of [5], which take a central role in this work.

\section{Terminology}

We will assume throughout that $R$ is a standard graded polynomial ring over an infinite field $k$. Given an ideal $I \subset R$ and an integer $r \geq 1$, the $r$ th symbolic power $I^{(r)}$ of $I$ is the contraction of $U^{-1} I^{r}$ under the natural homomorphism $R \rightarrow U^{-1} R$ of fractions, where $U$ is the complementary set of the union of the associated primes of $R / I$. In this work $I$ will be a codimension 2 perfect ideal, hence $R / I$ is Cohen-Macaulay and so $I$ is a pure (unmixed) ideal. In this setup then $I^{(r)}$

\footnotetext{
${ }^{0} 2010$ AMS Mathematics Subject Classification: 13A30, 13C14, 13C40, 13D02, 13E15, 13H10, 14E05, $14 \mathrm{E} 07$.

${ }^{1}$ Parts of this work were done while this author held a Doctoral Fellowship (CAPES, Brazil).

${ }^{2}$ Partially supported by a CNPq grant and a PVNS Fellowship (CAPES).
} 
is precisely the intersection of the primary components of the ordinary power $I^{r}$ relative to the associated primes of $R / I$, i.e., the unmixed part of $I^{r}$.

One says that $I$ is normally torsionfree provided $I^{(r)}=I^{r}$ for every $r \geq 1$.

We observe that, like the ordinary powers, the symbolic powers constitute a decreasing multiplicative filtration, so one can consider the corresponding symbolic Rees algebra $\mathcal{R}_{R}^{(I)}=$ $\bigoplus_{r \geq 0} I^{(r)} t^{r} \subset R[t]$. However, unlike the ordinary Rees algebra, this algebra may not be finitely generated over $R$.

Next is a review of the notion of the inversion factor associated to a Cremona map. This idea has been largely addressed in [10].

Let $k$ denote an arbitrary infinite field. A rational map $\mathfrak{G}: \mathbb{P}^{n-1} \rightarrow \mathbb{P}^{m-1}$ is defined by $m$ forms $\mathbf{g}=\left\{g_{1}, \ldots, g_{m}\right\} \subset R:=k[\mathbf{X}]=k\left[X_{1}, \ldots, X_{n}\right]$ of the same degree $d \geq 1$, not all null. We naturally assume throughout that $n \geq 2$. We often write $\mathfrak{G}=\left(g_{1}: \cdots: g_{m}\right)$ to underscore the projective setup and assume that $\operatorname{gcd}\left\{g_{1}, \cdots, g_{m}\right\}=1$ (in the geometric terminology, the linear system defining $\mathfrak{G}$ "has no fixed part"), in which case we call $d$ the degree of $\mathfrak{G}$.

We say that $\mathfrak{G}$ is a Cremona map if $m=n$ and $\mathfrak{G}$ is a birational map of $\mathbb{P}^{n-1}$. This means that there is a rational map $\mathbb{P}^{n-1} \rightarrow \mathbb{P}^{n-1}$ with defining coordinate forms $\mathbf{f}=\left\{f_{1}, \ldots, f_{n}\right\} \subset k[\mathbf{Y}]$ satisfying the relations

$$
\left(f_{1}(\mathbf{g}): \cdots: f_{n}(\mathbf{g})\right)=\left(X_{1}: \cdots: X_{n}\right),\left(g_{1}(\mathbf{f}): \cdots: g_{n}(\mathbf{f})\right)=\left(Y_{1}: \cdots: Y_{n}\right)
$$

The first of the above structural congruences

$$
\left(f_{1}\left(g_{1}, \ldots, g_{m}\right), \ldots, f_{n}\left(g_{1}, \ldots, g_{n}\right)\right) \equiv\left(X_{1}, \ldots, X_{n}\right)
$$

involving the inverse map gives a uniquely defined form $D \in R$ up to a nonzero scalar in $k$, such that $f_{i}\left(g_{1}, \ldots, g_{m}\right)=X_{i} D$, for every $i=1, \ldots, n$.

We call $D$ the source inversion factor of $\mathfrak{G}$

The classical theory of plane Cremona maps in characteristic zero relates the Jacobian of a homaloidal net with the principal curves of the corresponding Cremona map. In this connection the following general result has been proved in [10].

Proposition 1.1. $(\operatorname{char}(k)=0)$ Let $R=k\left[X_{1}, \ldots, X_{n}\right]$ be a polynomial ring over a field $k$ of characteristic zero, with its standard grading and let $\mathcal{L}=\left(\ell_{i j}\right)$ be an $n \times(n-1)$ matrix whose entries are linear forms in $R$. For every $i=1, \ldots, n$ write $\Delta_{i}$ for the signed $(n-1)$ minor of $\mathcal{L}$ obtained by omitting the $i$-th row and let $\Theta=\Theta(\boldsymbol{\Delta})$ denote the Jacobian matrix of $\Delta:=\left\{\Delta_{1}, \ldots, \Delta_{n}\right\}$.

If the ideal $I_{n-1}(\mathcal{L}):=(\boldsymbol{\Delta}) \subset R$ is of linear type then the rational map $\mathbb{P}^{n-1} \rightarrow \rightarrow \mathbb{P}^{n-1}$ defined by $\boldsymbol{\Delta}$ is a Cremona map and the associated source inversion factor is $\frac{1}{n-1} \operatorname{det}(\Theta)$.

\section{Main results}

Following common usage, one denotes by $I_{t}(\Psi) \subset R$ the ideal generated by the $t \times t$ minors of a matrix $\Psi$. 


\subsection{Generic catalectic matrices with leap}

The basic structure in this part is an $m \times(m-1) r$-leap catalecticant (or, shortly, $r$-catalecticant) in $R=k\left[X_{1}, \ldots, X_{n}\right]$, where $1 \leq r \leq m-1$ and $n=(m-1)(r+1)$ :

$$
\mathcal{C}=\left(\begin{array}{ccccc}
X_{1} & X_{2} & X_{3} & \ldots & X_{m-1} \\
X_{r+1} & X_{r+2} & X_{r+3} & \ldots & X_{m+r-1} \\
X_{2 r+1} & X_{2 r+2} & X_{2 r+3} & \ldots & X_{m+2 r-1} \\
\vdots & \vdots & \vdots & \ddots & \vdots \\
X_{(m-1) r+1} & X_{(m-1) r+2} & X_{(m-1) r+3} & \ldots & X_{(m-1) r+(m-1)}
\end{array}\right)
$$

The extreme values $r=1$ and $r=m-1$ yield, respectively, the ordinary Hankel matrix and the generic matrix.

A crucial property of $r$-catalectic matrices is essentially contained in [5]:

Proposition 2.1. An $r$-catalectic matrix of arbitrary size $v \times w(v \geq w)$ is 1-generic.

Proof. One can embed a catalectic matrix $\mathcal{C}$ such as the one above, of arbitrary size $v \times w$, as a submatrix of a $v^{\prime} \times w$ Hankel matrix for a suitable $v^{\prime} \geq v$. Namely

$$
\mathcal{C}=\left(\begin{array}{ccccc}
X_{1} & X_{2} & X_{3} & \ldots & X_{w} \\
X_{r+1} & X_{r+2} & X_{r+3} & \ldots & X_{r+w} \\
X_{2 r+1} & X_{2 r+2} & X_{2 r+3} & \ldots & X_{2 r+w} \\
\vdots & \vdots & \vdots & \ddots & \vdots \\
X_{(v-1) r+1} & X_{(v-1) r+2} & X_{(v-1) r+3} & \ldots & X_{(v-1) r+w}
\end{array}\right)
$$

can be augmented to a matrix $\mathcal{C}^{\prime}$ with the same number of columns $w$ by adding the "missing" Hankel blocks

$$
\mathcal{C}^{\prime}=\left(\begin{array}{ccccc}
X_{1} & X_{2} & X_{3} & \ldots & X_{w} \\
X_{r+1} & X_{r+2} & X_{r+3} & \ldots & X_{r+w} \\
X_{2 r+1} & X_{2 r+2} & X_{2 r+3} & \ldots & X_{2 r+w} \\
\vdots & \vdots & \vdots & \ddots & \vdots \\
X_{(v-1) r+1} & X_{(v-1) r+2} & X_{(v-1) r+3} & \ldots & X_{(v-1) r+w} \\
\hline X_{2} & X_{3} & X_{4} & \ldots & X_{w+1} \\
X_{3} & X_{4} & X_{5} & \ldots & X_{w+2} \\
\vdots & \vdots & \vdots & \ddots & \vdots \\
X_{r} & X_{r+1} & X_{r+2} & \ldots & X_{r+w-1} \\
\hline X_{r+2} & X_{r+3} & X_{r+4} & \ldots & X_{r+w+1} \\
X_{r+3} & X_{r+4} & X_{5} & \ldots & X_{r+w+2} \\
\vdots & \vdots & \vdots & \ddots & \vdots \\
X_{2 r} & X_{2 r+1} & X_{2 r+2} & \ldots & X_{2 r+w-1} \\
\hline \vdots & \vdots & \vdots & \ddots & \vdots
\end{array}\right)
$$


Now, given $A \in \mathrm{GL}_{k}(v)$ we consider the block matrix

$$
A^{\prime}=\left(\begin{array}{c|c}
A & \mathbf{0} \\
\mathbf{0} & \mathbf{1}_{v^{\prime}-v}
\end{array}\right)
$$

where $\mathbf{1}_{v^{\prime}-v}$ denotes the identity matrix os size $v^{\prime}-v$. Then $A^{\prime} \in \mathrm{GL}_{k}\left(v^{\prime}\right)$. This shows that if performing row operations on $\mathcal{C}^{\prime}$ produces no zero entry, then the same holds for $\mathcal{C}$. The same argument for column operations is trivial since we have not changed the column size of the original matrix.

Next, on $\mathcal{C}^{\prime}$ we move up the additional Hankel blocks in such a way so as to have the resulting matrix become a Hankel matrix of size $v^{\prime} \times w$. Note that this operation consists of iterated row permutations, so $\mathcal{C}^{\prime}$ is 1 -generic if and only the resulting matrix is. Since a Hankel matrix is 1-generic ([5, Proposition 4.2]), we are done.

Using this, we prove our result of this subsection:

Theorem 2.2. Let $I \subset R=k\left[X_{1}, \ldots, X_{n}\right]$ stand for the ideal of $(m-1)$-minors of an $m \times(m-1)$ $r$-leap catalectic matrix $\mathcal{C}$ as above, with $1 \leq r \leq m-1$. Then

(a) ht $\left(I_{t}(\mathcal{C})\right) \geq m-t+2$ for $1 \leq t \leq m-2$ and ht $(I)=2$.

(b) $R / I$ is a Cohen-Macaulay normal domain.

(c) I is an ideal of linear type.

(d) I is normally torsionfree.

Proof. (a) The result is clear for $t=1$, hence assume that $2 \leq t \leq m-1$. For $t$ in this interval, consider the submatrix $[\mathcal{C}]_{t}$ of $\mathcal{C}$ formed by its first $t$ columns. By Proposition $2.1,[\mathcal{C}]_{t}$ is 1-generic, hence its ideal $I_{t}\left([\mathcal{C}]_{t}\right)$ of $t$-minors (maximal minors) of $[\mathcal{C}]_{t}$ is prime and satisfies ht $\left(I_{t}\left([\mathcal{C}]_{t}\right)\right) \geq m-1-t+2=m-t+1$ (cf. [5, Theorem 2.1]).

To conclude, it suffices to show that the inclusion $I_{t}\left([\mathcal{C}]_{t}\right) \subset I_{t}(\mathcal{C})$ is proper for $t \leq m-2$. For this, let $\Delta$ stand for the lower rightmost $t$-minor of $\mathcal{C}$. Since $\Delta$ has a term involving effectively the last variable, while no $t$-minor minor of $[\mathcal{C}]_{t}$ (with $t \leq m-2$ ) has such a term, and since all minors in consideration live in the same degree, we clearly have $\Delta \notin I_{t}\left([\mathcal{C}]_{t}\right)$.

(b) Cohen-Macaulayness is obvious. Since $\mathcal{C}$ is 1-generic then every prime $Q \subset R / I$ such that $(R / I)_{Q}$ is not regular must contain the ideal $I_{m-2}(\mathcal{C}) / I$ ([5, Corollary 3.3]). Then ht $(Q) \geq$ ht $\left(I_{m-2}(\mathcal{C}) / I\right) \geq 4-2=2$, by (a). Therefore, $R / I$ satisfies the Serre condition $\left(R_{1}\right)$, hence $R / I$ is a normal domain.

(c) The estimates in (a) imply that $I$ satisfies the condition $\left(F_{1}\right)$ (or $G_{\infty}$ ). Therefore, it is of linear type (see [7]).

(d) The assertion could possibly be derived from the methods of [9], but one can give a direct argument in the present situation. By part (c), $I$ is of linear type. Since $I$ is strongly CohenMacaulay ([1, Theorem 2.1(a)]) then the Rees algebra of $I$ is Cohen-Macaulay ([7, Theorem 9.1]), and hence so is the associated graded ring of $I$. On the other hand, by part (b) the ideal $I$ is prime. By [6, Proposition $3.2(1)$ ], the assertion is equivalent to having

$$
\ell_{P}(I) \leq \max \{\text { ht } P-1, \text { ht } I\},
$$


for every prime ideal $P \supset I$. We may assume that ht $P \geq 3$ since $I$ is a height 2 prime. Therefore, we have to show that $\ell_{P}(I) \leq$ ht $P-1$. If $P=(\mathbf{X})$ the result is clear since $\ell_{(\mathbf{X})} \leq$ $\mu(I)=m \leq n-1=\mathrm{ht}(\mathbf{X})-1$. Therefore, we may assume that $P \subsetneq(\mathbf{X})$, hence ht $P \leq n-1$. In particular, $I_{1}(\mathcal{C}) \not \subset P$, so consider the index $t_{0}:=\max \left\{1 \leq s \leq m-2 \mid I_{s}(\mathcal{C}) \not \subset P\right\}$. Pick a $t_{0}$-minor $\Delta$ of $\mathcal{C}$ not contained in $P$, so that, in particular, $R_{P}$ is a localization of the ring of fractions $R_{\Delta}=R\left[\Delta^{-1}\right] \subset k(\mathbf{X})$. By a standard row-column elementary operation procedure, there is an $\left(m-t_{0}\right) \times\left(m-t_{0}-1\right)$ matrix $\widetilde{\mathcal{C}}$ over $R_{P}$ such that $I_{P}=I_{m-1-t_{0}}(\widetilde{\mathcal{C}})$.

Now, we have ht $I_{t_{0}+1}(\mathcal{C})-1 \geq m-t_{0}$ from item (a) and, since $I_{t_{0}+1}(\mathcal{C}) \subset P$ by definition of $t_{0}$, it follows that $m-t_{0} \leq \mathrm{ht} P-1$. Therefore

$\ell_{P}(I)=\ell\left(I_{m-1-t_{0}}(\widetilde{\mathcal{C}})\right) \leq \min \left\{\mu\left(I_{m-1-t_{0}}(\widetilde{\mathcal{C}})\right)\right.$, ht $\left.P\right\}=\min \left\{m-t_{0}\right.$, ht $\left.P\right\} \leq$ ht $P-1$.

\subsection{Generic sub-Hankel matrices}

In this part we consider a degeneration of the generic Hankel (i.e., 1-catalectic) matrix $\mathcal{H}$, in which a lower corner of suitable size has its entries replaced by zeros. A version of this model for square matrices has been introduced in [2] (see also [8]) in connection to the construction of homaloidal determinants.

Let $R=k\left[X_{1}, \ldots, X_{n}\right]$ and let $m$ be such that $4 \leq m+1 \leq n \leq 2(m-1)$. Set

$$
\mathcal{S H}=\left(\begin{array}{ccccccccc}
X_{1} & \ldots & X_{n-m+1} & X_{n-m+2} & X_{n-m+3} & X_{n-m+4} & \ldots & X_{m-2} & X_{m-1} \\
X_{2} & \ldots & X_{n-m+2} & X_{n-m+3} & X_{n-m+4} & X_{n-m+5} & \ldots & X_{m-1} & X_{m} \\
\vdots & & \vdots & \vdots & \vdots & \vdots & & \vdots & \vdots \\
X_{n-m+2} & \ldots & X_{2 n-2 m+2)} & X_{2 n-2 m+3} & X_{2 n-2 m+4} & X_{2 n-2 m+5} & \ldots & X_{n-1} & X_{n} \\
\hline X_{n-m+3} & \ldots & X_{2 n-2 m+3} & X_{2 n-2 m+4} & X_{2 n-2 m+5} & X_{2 n-2 m+6} & \ldots & X_{n} & 0 \\
X_{n-m+4} & \ldots & X_{2 n-2 m+4} & X_{2 n-2 m+5} & X_{2 n-2 m+6} & X_{2 n-2 m+7} & \ldots & 0 & 0 \\
\vdots & & \vdots & \vdots & \vdots & \vdots & & \vdots & \vdots \\
X_{m-1} & \ldots & X_{n-1} & X_{n} & 0 & 0 & \ldots & 0 & 0 \\
X_{m} & \ldots & X_{n} & 0 & 0 & 0 & \ldots & 0 & 0
\end{array}\right)
$$

Note that $n=2(m-1)$ is the case of the ordinary Hankel matrix. This model has the following properties:

Theorem 2.3. Let $m \geq 3$ and $n \leq 2(m-1)$. Set $I:=I_{m-1}(\mathcal{S H})$. Then:

(a) ht $\left(I_{t}(\mathcal{S H})\right) \geq m-t+2$ for $1 \leq t \leq m-2$ and $I$ is a height 2 prime ideal.

(b) $R / I$ is normal if and only if $n \geq m+2$

(c) I is an ideal of linear type.

(d) I is normally torsionfree.

Proof. (a) The proof is similar to the argument used in the case of the catalecticant, but there are differences due to the presence of zeros. As before, the case $t=1$ is immediate. Next we let $[\mathcal{S H}]_{t}$ denote the submatrix of $\mathcal{S H}$ with the first $t$ columns, for values of $t$ in the 
range $2 \leq t \leq m-2$. For $t \leq n-m+1$ the matrix $[\mathcal{S H}]_{t}$ is an ordinary $m \times t$ Hankel matrix. Therefore, it is 1 -generic, hence its $t$-minors (maximal minors) generate a prime ideal of codimension $\geq m-t+1$ (also directly by the observation after [5, Proposition 4.3]). For $t>n-m+1$, one has as follows

$$
k\left[X_{1}, \ldots, X_{n}\right] / I_{t}\left([\mathcal{S H}]_{t}\right) \simeq k\left[X_{1}, \ldots, X_{n}, X_{n+1}, \ldots, X_{n+1+s}\right] /\left(X_{n+1}, \ldots, X_{n+1+s}, I_{t}\left([\mathcal{H}]_{t}\right)\right),
$$

where $s=t-(n-m+1)$ and $[\mathcal{H}]_{t}$ is the Hankel matrix of size $m \times t$. Since $s \leq m-2$ (because $t<n)$ then we can use [4, Theorem 1] to deduce again that $I_{t}\left([\mathcal{S H}]_{t}\right)$ is a prime ideal of codimension $m-t+1$. The second assertion of (a) can be deduced similarly.

Now, to complete the argument it suffices to show that the inclusion $I_{t}\left([\mathcal{S H}]_{t}\right) \subset I_{t}(\mathcal{S H})$ is proper for $2 \leq t \leq m-2$, since the first of these ideals is prime. This is clear if $t \leq 2(m-1)-n$ and $2(m-1)>n$ since a non-trivial power of $X_{n}$ is a $t$-minor in $I_{t}(\mathcal{S H})$ that cannot lie in the prime $I_{t}\left([\mathcal{S H}]_{t}\right)$ for $t \geq 2$. Thus, assume that $t>2(m-1)-n$. The argument is akin to the one used in the proof of the catalectic case. Namely, let $\Delta$ stand for the lower-rightmost $t$-minor of $\mathcal{S H}$

$$
\left(\begin{array}{ccccccc}
X_{n-(t-r)-(t-1)} & X_{n-(t-r)-(t-1)+1} & \ldots & X_{n-(t-1)} & \ldots & X_{n-(t-r)-1} & X_{n-(t-r)} \\
X_{n-(t-r)-(t-1)+1} & X_{n-(t-r)-(t-1)+2} & \ldots & X_{n-(t-1)+1} & \ldots & X_{2 m-t} & X_{n-r} \\
\vdots & \vdots & \ddots & \vdots & \ddots & \vdots & \\
X_{n-(t-1)} & X_{n-(t-1)+1} & \ldots & X_{n-(t-1)+(t-r)} & \ldots & X_{n-1} & X_{n} \\
X_{n-(t-1)+1} & X_{n-(t-1)+2} & \ldots & X_{n-(t-1)+(t-r)+1} & \ldots & X_{n} & 0 \\
\vdots & \vdots & \ddots & \vdots & \ddots & \vdots & \vdots \\
X_{n-(t-r)} & X_{n-(t-r)+1} & \ldots & X_{n} & \ldots & 0 & 0
\end{array}\right)
$$

where $r=2(m-1)-n$, which is the number of times $X_{n}$ appears on the original matrix. By Laplace along the first row, it obtains

$$
\Delta=X_{n-(t-r)-(t-1)}\left|\begin{array}{cccccc}
X_{n-(t-r)-(t-1)+2} & \ldots & X_{n-(t-1)+1} & \ldots & X_{2 m-t} & X_{n-r} \\
\vdots & \vdots & \ddots & \vdots & \ddots & \vdots \\
X_{n-(t-1)+1} & \ldots & X_{n-(t-1)+(t-r)} & \ldots & X_{n-1} & X_{n} \\
X_{n-(t-1)+2} & \ldots & X_{n-(t-1)+(t-r)+1} & \ldots & X_{n} & 0 \\
\vdots & \ddots & \vdots & \ddots & \vdots & \vdots \\
X_{n-(t-r)+1} & \ldots & X_{n} & \ldots & 0 & 0
\end{array}\right|+H
$$

where $H$ does not involve $X_{n-(t-r)-(t-1)}$ as the latter occurs only once on the matrix.

Now, by induction the determinant multiplying $X_{n-(t-r)-(t-1)}$ in this expression is of the form $G \cdot X_{n}^{r}$, with $G$ a nonzero polynomial of degree $(t-1)-r$. This entails:

$$
\Delta=\left(X_{n-(t-r)-(t-1)} G+F\right) X_{n}^{r}+\left(\text { terms of degree less than } r \text { in } X_{n}\right)
$$

where $F$ (possibly vanishing) comes from terms in $H$, hence, in particular, does not involve $X_{n-(t-r)-(t-1)}$. From this, $X_{n-(t-r)-(t-1)} G+F \neq 0$. On the other hand, any $t \times t$ submatrix of $\mathcal{S H}_{t}$ has at most $r-1$ entries equal to $X_{n}$. It follows that the $X_{n}$-degree on any $t$-minor generating $I_{t}\left(\mathcal{S H}_{t}\right)$ is strictly less than $r$.

Thus, we are led to conclude that $\Delta \notin I_{t}\left(\mathcal{S H}_{t}\right)$, as claimed.

(b) We first show that $I$ does not satisfy $\left(R_{1}\right)$ if $n=m+1$ (the lowest possible value). For this, consider the height 3 prime $P=\left(X_{n-2}, X_{n-1}, X_{n}\right)$. Clearly, $I \subset P$ by direct inspection on 
the shape of the matrix. Note that the upper left $(n-3)$-minor of $\mathcal{S H}$ has the form $X_{n-3}^{n-3}+q$ where $q \in P$, hence does not belong to $I$. After appropriate row/column operations, we se that $I_{P}=\left(\Delta_{n-2}, \Delta_{n-1}\right)$, where $\Delta_{i}$ denotes $(n-2)$-minor of $\mathcal{S H}$ obtained by omitting the $i$ th row. We claim that $R_{P} / I_{P}$ is not regular. For this, it suffices to show that $\Delta_{n-2} \in P^{2}$. But

$$
\Delta_{n-2}=(-1)^{n-1} X_{n-1} \operatorname{det}\left(\begin{array}{cccc}
X_{2} & X_{3} & \ldots & X_{n-2} \\
X_{3} & X_{4} & \ldots & X_{n-1} \\
\vdots & \vdots & \vdots & \vdots \\
X_{n-2} & X_{n-1} & \ldots & 0
\end{array}\right)+(-1)^{n} X_{n} \operatorname{det}\left(\begin{array}{cccc}
X_{1} & X_{3} & \ldots & X_{n-2} \\
X_{2} & X_{4} & \ldots & X_{n-1} \\
\vdots & \vdots & \vdots & \vdots \\
X_{n-3} & X_{n-1} & \ldots & 0
\end{array}\right)
$$

Note that the two determinants have same last column and the nonzero entries on the column are $X_{n-2}, X_{n-1}, X_{n}$. Therefore, expanding these determinants along their last column clearly shows the claim.

Conversely, suppose now that $n \geq m+2$. We will use use a result of [5]. For this, first switch to the transpose $M$ of $\mathcal{S H}$ in order to conform with the notation in [5]. Next let $M^{\prime}$ denote the transpose of the Hankel matrix of size $(m-1) \times m$. Thinking of $M \subset M^{\prime}$ as the respective $k$ subspaces spanned by the entries, one has $\operatorname{codim}_{M^{\prime}} M=2(m-1)-n$. Our hypothesis implies that $\operatorname{codim}_{M^{\prime}} M \leq m-4$. Now, $M^{\prime}$ is 1-generic, hence the singular locus of $\operatorname{Proj}(R / I)$ is contained in the union of $\operatorname{Proj}\left(R / I_{m-2}(\mathcal{S H})\right)$ and a certain set of codimension at least $m-2-\operatorname{codim}_{M^{\prime}} M$ in $\operatorname{Proj}(R / I)$ - according to the discussion immediately following the statement of [5, Theorem 2.1 (3)] and its proof in [5. Proposition 3.1 and The completion of the proof of Theorem 2.1]. The first of these two has codimension $\geq 2$ in $\operatorname{Proj}(R / I)$ by the estimates of item (a). As for the second, its codimension in $\operatorname{Proj}(R / I)$ is now at least $m-2-\operatorname{codim}_{M^{\prime}} M \geq m-2-(m-4)=2$. This shows that $R / I$ satisfies Serre's property $\left(R_{1}\right)$, hence is normal.

(c) and (d) are proved exactly the same way as in Theorem 2.2

\section{$2.3 \quad$ Semi-Hankel matrices}

Let again $n \leq 2(m-1)$, this time around with $3 \leq m \leq n$, thus allowing for the equality $n=m$ (while $n \geq m+1$ was stipulated in the sub-Hankel case.) The model of this part is in a sense an ancestral of the previous sub-Hankel model, in which one specializes certain entries of the $m \times(m-1)$ generic Hankel matrix to a few independent linear forms:

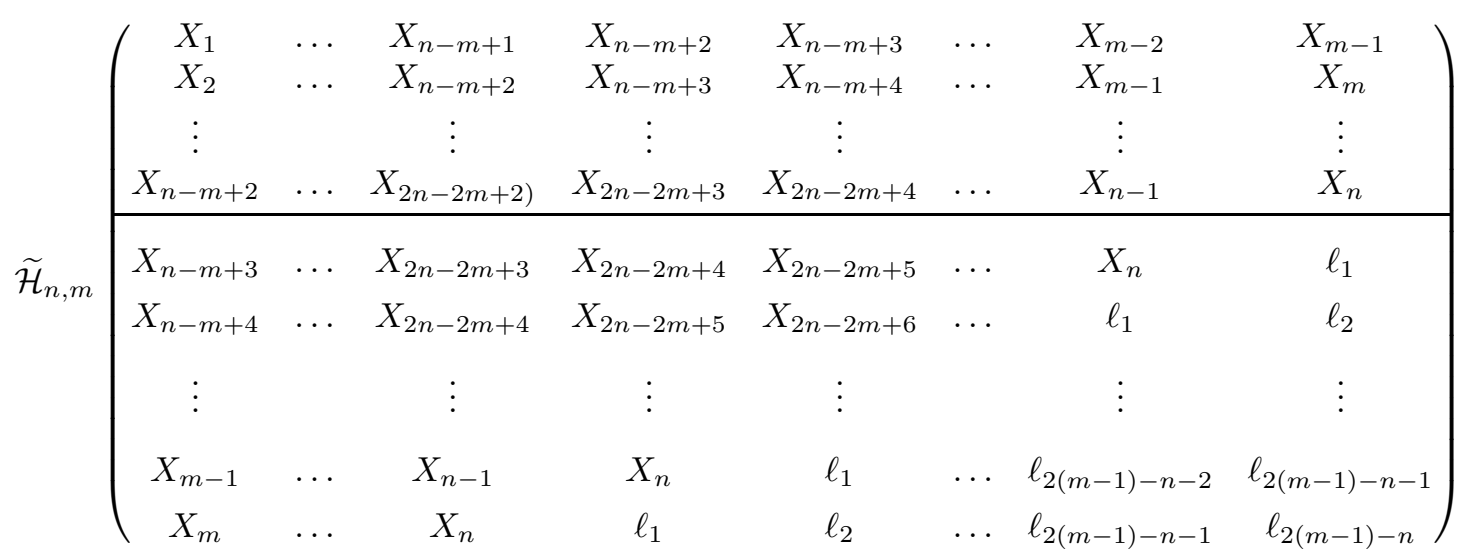


where $\left\{\ell_{1}, \ell_{2}, \ldots, \ell_{2(m-1)-n-1}, \ell_{2(m-1)-n}\right\}$ are independent linear forms in $k\left[X_{1}, \ldots, X_{n}\right]$, an assumption that makes sense since $2(m-1)-n \leq n$.

Theorem 2.4. Let $I:=I_{n-1}\left(\widetilde{\mathcal{H}}_{n, m}\right)$. Then:

(a) $\operatorname{ht}\left(I_{t}\left(\widetilde{\mathcal{H}}_{n, m}\right)\right) \geq m-t+2$ in the range $1 \leq t \leq m-2$, while ht $(I)=2$

(b) $R / I$ is a normal domain.

(c) I is an ideal of linear type.

(d) $(\operatorname{char}(k)=0)$ For every $r \geq 0$ such that $I^{(r)} \neq I^{r}$, the $R$-module $I^{(r)} / I^{r}$ is $(\mathbf{X})$-primary.

Proof. (a) We use same strategy as before, by considering the submatrix $\left[\widetilde{\mathcal{H}}_{n, m}\right]_{t}$ of $\widetilde{\mathcal{H}}_{n, m}$ formed with the first $t \leq m-1$ columns. Since this matrix specializes from the full generic $m \times t$ Hankel matrix - which is 1-generic - modulo a regular sequence of 1-forms of cardinality $t-(n-m+1) \leq t-2$, we can apply [5, Theorem 2.1 and Corollary 3.3], by which one has the following properties:

(i) ht $I_{t}\left(\left[\widetilde{\mathcal{H}}_{n, m}\right]_{t}\right)=m-t+1$, for $2 \leq t \leq m-1$.

(ii) $I_{t}\left(\left[\widetilde{\mathcal{H}}_{n, m}\right]_{t}\right)$ is a prime ideal.

(iii) The ideal $I_{t-1}\left(\left[\widetilde{\mathcal{H}}_{n, m}\right]_{t}\right) / I_{t}\left(\left[\widetilde{\mathcal{H}}_{n, m}\right]_{t}\right)$ defines the singular locus of $R[\mathbf{Z}] / I_{t}\left(\left[\widetilde{\mathcal{H}}_{n, m}\right]_{t}\right)$

Consider the $t$-minor $\Delta$ of the lower rightmost corner of $\widetilde{\mathcal{H}}_{n, m}$. The argument is the same as of the proof of Theorem 2.3 (a): we may assume that $t \geq 2(m-1)-n$, hence $\Delta$ has a leading term in $X_{n}$ coming from the anti-diagonal with $X_{n}$ 's throughout - note that the terms involving the linear forms can only have a smaller degree in $X_{n}$. Again, we are led to conclude by the same token as before that $\Delta \notin I_{t}\left(\left[\widetilde{\mathcal{H}}_{n, m}\right]_{t}\right)$ for $2 \leq t \leq m-2$.

This proves that ht $\left(I_{t}\left(\widetilde{\mathcal{H}}_{n, m}\right)\right) \geq m-t+2$ in the range $1 \leq t \leq m-2$, and also that ht $(I)=2$.

(b) Using the result of (iii) in the case of $t=m-1$, we know that the singular locus of $R / I$ is defined by the ideal $I_{m-2}\left(\widetilde{\mathcal{H}}_{n, m}\right) / I_{m-1}\left(\widetilde{\mathcal{H}}_{n, m}\right)$. By (a) the latter has codimension $\geq m-(m-2)+2-2=2$, hence $R / I$ has the property $\left(R_{1}\right)$. Therefore, $R / I$ is a (CohenMacaulay) normal domain.

(c) As previously remarked, (a) implies condition $\left(F_{1}\right)$. Since $R / I$ is Cohen-Macaulay of codimension $2, I$ is of linear type.

(d) Fixing an $r \geq 0$, suppose that $I^{(r)} / I^{r} \neq\{0\}$. The assertion is equivalent to saying that a power of $(\mathbf{X})$ annihilates $I^{(r)} / I^{r}$ i.e., that $I^{(r)}{ }_{P}=I^{r}{ }_{P}$ for every prime $P \neq(\mathbf{X})$. Letting $r \geq 0$ run, this is in turn equivalent to claiming that the associated graded ring $\operatorname{gr}_{I}(R)$ is torsionfree over $R / I$ locally on the punctured spectrum $\operatorname{Spec}(R) \backslash(\mathbf{X})$.

Thus, let $P \neq(\mathbf{X})$ be a prime containing $I$. By (a), as already pointed out, $I$ and hence, also, $I_{P}$ satisfies the condition $\left(F_{1}\right)$. As in the proof of Theorem $2.2(\mathrm{~d})$, we know that the associated graded ring $\operatorname{gr}_{I_{P}}\left(R_{P}\right)$ is Cohen-Macaulay. Therefore, by the same token and since ht $I=2$, one has to show the local estimates

$$
\ell_{Q}(I)=\ell_{Q_{P}}\left(I_{P}\right) \leq \mathrm{ht}\left(Q_{P}\right)-1=\mathrm{ht} Q-1
$$


for every prime $Q \subset P$.

Fixing such a prime $Q$, set $t_{0}:=\max \left\{1 \leq s \leq m-2 \mid I_{s}\left(\widetilde{\mathcal{H}}_{n, m}\right) \not \subset Q\right\}$ - again $t_{0}$ makes sense since $I_{1}\left(\widetilde{\mathcal{H}}_{n, m}\right) \not \subset Q$. Trading $Q$ for $P$ in the proof of Theorem 2.2 (d), the rest of the argument is literally the same.

In the case where $n=m$, we gather some geometric information:

Proposition 2.5. Let $I:=I_{n-1}\left(\widetilde{\mathcal{H}}_{n, n}\right)$. Then:

(i) $I^{(\ell)}=I^{\ell}$ for $1 \leq \ell \leq n-2$

(ii) The rational map $\mathfrak{G}: \mathbb{P}^{n-1} \rightarrow \mathbb{P}^{n-1}$ defined by the $(n-1)$-minors of $\widetilde{\mathcal{H}}_{n, n}$ is a Cremona map.

(iii) $(\operatorname{char}(k)=0)$ The symbolic Rees algebra $\mathcal{R}^{(I)}$ of I is a Gorenstein normal domain such that $\mathcal{R}^{(I)}=R\left[I t, D t^{n-1}\right]$, where $D$ is the source inversion factor of the Cremona map defined by the $(n-1)$-minors of $\widetilde{\mathcal{H}}_{n, n}$; moreover, $D$ coincides with the Jacobian determinant of the $(n-1)$-minors of $\widetilde{\mathcal{H}}_{n, n}$.

Proof. (i) By Theorem 2.4 (c), $I$ is an ideal of linear type; in particular, it satisfies property $\left(G_{\infty}\right)$, i.e., $\mu\left(I_{p}\right) \leq$ ht $P$ for every prime $P \subset R$. According to [14, Theorem 5.1], under this condition, for every $1 \leq \ell \leq n-1$ the complex

$$
\mathcal{K}_{\ell}: 0 \rightarrow F_{\ell} \rightarrow F_{\ell-1} \rightarrow \ldots \rightarrow F_{1} \rightarrow F_{0} \rightarrow 0
$$

is a free resolution of $I^{\ell}$, where $F_{i}:=\bigwedge^{i} R^{n-1} \otimes_{R} \mathcal{S}_{(n-1)-i}\left(R^{n}\right)$ and $d: F_{i} \rightarrow F_{i-1}$ is defined through

$$
d\left(e_{1} \wedge \ldots \wedge e_{i} \otimes g\right):=\sum_{l=1}^{i} e_{1} \wedge \ldots \wedge \widehat{e}_{l} \wedge \ldots \wedge e_{i} \otimes \varphi\left(e_{l}\right) g .
$$

It follows that, in the range $1 \leq \ell \leq n-2$, the $R$-module $\left(R / I^{\ell}\right)$ has homological dimension at most $n-1$, hence $(\mathbf{X})$ is not an associated prime thereof. By induction using Ass $\left(R / I^{\ell}\right) \subset$ $\operatorname{Ass}\left(I^{(\ell)} / I^{\ell}\right) \cup \operatorname{Ass}\left(R / I^{(\ell)}\right)$ and drawing upon Theorem 2.4, we are done.

(ii) This follows from Theorem 2.4 (a) and (c) via [3, Proposition 3.4].

(iii) The symbolic Rees algebra $\mathcal{R}^{(I)}$ of $I$ is a Gorenstein ring; indeed, it is a quasi-Gorenstein Krull domain since $I$ is a codimension 2 prime ideal ([12]). On the other hand, by the proof of [13, Corollary $3.4(\mathrm{~b})], \mathcal{R}^{(I)}$ is finitely generated since one has an isomorphism $\mathcal{R}^{(I)} \simeq \mathcal{R}(I)\left[t^{-1}\right]=$ $R\left[I t, t^{-1}\right]$. Moreover, the latter is Cohen-Macaulay since $\mathcal{R}(I)$ is Cohen-Macaulay - same argument as in the proof of [10, Proposition $2.9(\mathrm{~b})]$. It follows that $\mathcal{R}^{(I)}$ is a Gorenstein normal domain and that it is generated over the Rees algebra $R[I t]$ by one single element. It remains to identify this element in the form $D t^{d}$, for some $D \in R$ and a uniquely determinde exponent $d$.

To go about this, we use (ii), namely, let $\mathfrak{d}_{1}, \ldots, \mathfrak{d}_{n} \in k[\mathbf{Y}]$ be forms of the same degree, with gcd $=1$, defining the inverse map and let $D \in R$ denote the corresponding source inversion factor. Write $J=\left(\mathfrak{d}_{1}, \ldots, \mathfrak{d}_{n}\right) \subset k[\mathbf{Y}]$. By definition, one has

$$
D=\mathfrak{d}_{i}\left(\Delta_{1}, \ldots, \Delta_{n}\right) / X_{i}, 1 \leq i \leq n,
$$

where $\boldsymbol{\Delta}:=\left\{\Delta_{1}, \ldots, \Delta_{n}\right\}$ are the (signed) minors generating $I$. But under a Cremona map, the two Rees algebras $\mathcal{R}_{R}(I)=R[I t] \subset R[t]$ and $\mathcal{R}_{k[\mathbf{Y}]}(J)=k[\mathbf{Y}][J u] \subset k[\mathbf{Y}][u]$ get identified by a 
$k$-isomorphism that maps $Y_{i} \mapsto \Delta_{i} t$ and $X_{i} \mapsto \mathfrak{d}_{i} u$ (see. e.g., [11, Proposition 2.1]). Then $D$ is identified with $\mathfrak{d}_{1} / X_{1}$ in the common field of fractions. Using (i) above, the symbolic algebra is generated by $I t$ and $D t^{n-1}$ as a consequence of [15, Corollary 7.4.3 (b)].

The additional statement reads out of Proposition 1.1.

\section{References}

[1] L. Avramov and J. Herzog, The Koszul algebra of a codimension 2 embedding, Math. Z. 175 (1980) 249-260.

[2] C. Ciliberto, F. Russo and A. Simis, Homaloidal hypersurfaces and hypersurfaces with vanishing Hessian, Advances in Math., 218 (2008) 1759-1805.

[3] A. V. Dória, H. Hassanzadeh and A. Simis, A chracteristic free criterion of birationality, Advances in Math., 230 (2012), 390-413.

[4] D. Eisenbud, On the resiliency of determinantal ideals, Proceedings of the U.S.-Japan Seminar, Kyoto 1985. In Advanced Studies in Pure Math. II, Commutative Algebra and Combinatorics, ed. M. Nagata and H. Matsumura, North-Holland (1987) 29-38.

[5] D. Eisenbud, Linear sections of determinantal varieties, Amer. J. Mathematics, 110 (1988), $541-575$.

[6] D. Eisenbud and C. Huneke, Cohen-Macaulay Rees algebras and their specializations, J. Algebra 81 (1983), 202-224.

[7] J. Herzog, A. Simis and W. V. Vasconcelos, Koszul homology and blowing-up rings, Commutative Algebra, Lecture Notes in Pure and Applied Math., Vol. 84, 79-169, Marcel-Dekker, New York, 1983.

[8] M. Mostafazadehfard and A. Simis, Homaloidal determinants, ongoing.

[9] L. D. Nam, The determinantal ideals of extended Hankel matrices, J. Pure Appl. Algebra 215 (2011), 1502-1515.

[10] Z. Ramos and A. Simis, Symbolic powers of perfect ideals of codimension 2 and birational maps, J. Algebra, to appear.

[11] A. Simis, Cremona transformations and some related algebras, J. Algebra 280 (2004), $162-179$.

[12] A. Simis and N. V. Trung, Divisor class group of ordinary and symbolic blow-ups, Math. Z. 198 (1988), 479-491.

[13] A. Simis, B. Ulrich and W. Vasconcelos, Jacobian dual fibrations, Amer. J. Math. 115 (1993), 47-75.

[14] A. B. Tchernev, Torsion freeness of symmetric powers of ideals, Trans. Amer. Math. Soc., 359 (2007), 3357-3367. 
[15] W. Vasconcelos, Arithmetic of Blowup Algebras, London Mathematical Society, Lecture Notes Series 195, Cambridge University Press, 1994.

\section{Authors' addresses:}

Zaqueu Ramos, Departamento de Matemática, CCET, Universidade Federal de Sergipe 49100-000 São Cristovão, Sergipe, Brazil

e-mail: zaqueu.ramos@gmail.com

Aron Simis, Departamento de Matemática, CCEN, Universidade Federal de Pernambuco 50740-560 Recife, PE, Brazil.

e-mail: aron@dmat.ufpe.br 